\begin{tabular}{|c|l|}
\hline Title & Near-infrared spectroscopic study of water at high temperatures and pressures \\
\hline Author(s) & Jin, Y usuke; Ikawa, Shun-ichi \\
\hline Citation & $\begin{array}{l}\text { The Journal of Chemical Physics, 119(23), 12432-12438 } \\
\text { https://doi.org/10.1063/1.1628667 }\end{array}$ \\
\hline Issue Date & 2003 \\
\hline Doc URL & http://hdl.handle.net/2115/1396 \\
\hline Rights & Copyright $\odot 2003$ A merican Institute of Physics \\
\hline Type & article \\
\hline File Information & JCP119-23.pdf \\
\hline
\end{tabular}

Instructions for use 


\title{
Near-infrared spectroscopic study of water at high temperatures and pressures
}

\author{
Yusuke Jin and Shun-ichi lkawa \\ Division of Chemistry, Graduate School of Science, Hokkaido University, Sapporo, 060-0810, Japan
}

(Received 10 July 2003; accepted 30 September 2003)

\begin{abstract}
Near-infrared absorption of the $\mathrm{OH}$ stretching overtone transition of water has been measured at temperatures and pressures in the ranges of 373-673 $\mathrm{K}$ and $20-400$ bar, respectively. The absorption profile at $673 \mathrm{~K}$ and 400 bar retains a mark of rotational structure, indicating that an appreciable proportion of water molecules can rotate quite freely. The molar absorption intensity decreases linearly with increasing pressure in the low-pressure region. Enthalpy for dimerization has been estimated to be $15 \pm 3 \mathrm{~kJ} / \mathrm{mol}$ from the temperature dependence of the slopes. Plots of the molar absorption intensity against molar concentration are observed to be located on a single curve irrespective of the temperature. This fact indicates that the ratio of hydrogen-bond formation is largely dependent on the molar density only. A good correlation between the molar absorption intensity and the first moments of the band has been found out; this will be useful in the study of aqueous mixtures. (C) 2003 American Institute of Physics. [DOI: 10.1063/1.1628667]
\end{abstract}

\section{INTRODUCTION}

Water at high temperatures and pressures exhibits remarkably different properties from ambient water. It becomes a good solvent for nonpolar compounds such as hydrocarbons, ${ }^{1-3}$ which are usually thought of as hydrophobic substances. In contrast, high-temperature water is a poor solvent for ionic substances due to dehydration of the ions, which facilitates ion-ion association. ${ }^{4-6}$ This change in the solvent property of water is explained by a decrease in the static dielectric constant. For example, this value decreases from 78.41 at $298 \mathrm{~K}$ and 1 bar to 20.22 at $573 \mathrm{~K}$ and $100 \mathrm{bar}$, and 11.95 at $648 \mathrm{~K}$ and 300 bar. $^{7}$ The solubility of water in hydrocarbons also increases with increasing temperature under pressure; water and hydrocarbons become completely miscible above a certain temperature and pressure, in any ratio. For example, water and benzene form a homogeneous mixture at temperatures and pressures above $570 \mathrm{~K}$ and 200 bar. ${ }^{1-3}$ The dynamical property also exhibits remarkable temperature-dependent change, and molecular diffusion coefficients significantly increase with increasing temperature. ${ }^{8,9}$ The notably large mutual solubility of water and organic compounds and accelerated molecular diffusion make high-temperature water a promising medium for chemical reactions. ${ }^{10,11}$ In addition, high-temperature water may sometimes act as a reactant or catalyst, which can be explained by the fact that the ion product of water is a few orders of magnitude higher than that of water under ambient condition. ${ }^{11-13}$ High-temperature water has attracted considerable attention as a reaction medium because of these properties, particularly for environmentally benign chemical processes. It is of particular importance for environmentprotecting technology where hydrothermal reactions are applied to the destruction of toxic waste chemicals. ${ }^{12-17}$ Water-hydrocarbon mixtures at high temperatures and pressures are also important in a wide range of industrial situations. The physicochemical properties of the mixtures are indispensable to the design and maintenance of plants for oil refineries, the gas industry, and the petrochemical industry. ${ }^{18-21}$

The temperature dependence of the solubility phenomena of aqueous systems mentioned above is thought to be related to a change in water structure, the hydrogen bond network, at elevated temperatures. Intensified thermal molecular motions will loosen or destroy the hydrogen-bonded structure and reduce orientational correlation among water molecules, thus causing a decrease in the dielectric constant. Such a structural loosening also makes room for the acceptance of even hydrophobic hydrocarbons, and in addition, facilitates the transfer of water to the hydrocarbon phase, i.e., reduces the chemical potential difference for water molecules between the two phases. In addition to the solubility phenomena, molecular mobility increases as the hydrogenbonded structure loosens, which results in a remarkable increase in the diffusion coefficients.

In order to understand the characteristic features of hightemperature water, it is essential to examine how the hydrogen-bonded structure of water varies with temperature and pressure. Various experimental methods have been applied to date to investigate the hydrogen-bond network of high temperature water, particularly at supercritical conditions. Neutron-scattering ${ }^{22-24}$ and $\mathrm{x}$-ray ${ }^{25,26}$ studies have provided configurational information on a local average structure, while NMR, ${ }^{27-29}$ infrared, ${ }^{30-34}$ and $\operatorname{Raman}^{35-38}$ spectroscopy have revealed how the degree of hydrogen bonding varies with temperature and pressure. The static dielectric constant has also been used to discuss water structure up to the critical point. ${ }^{39}$ Molecular dynamics (MD) simulations have performed an important role in the investigation of the structural change of water over a wide range of temperatures and pressures. ${ }^{40-49}$ Significant discrepancies between simulations and experimental results have sometimes occurred, which suggested that corrections were required for 
the interpretation of experimental results. ${ }^{43,44}$ Meanwhile, various devices to improve the simulation of water have been proposed. ${ }^{49,50}$

It is now commonly accepted that hydrogen bonds persist at temperatures even higher than the critical temperature of water. However, the quasitetrahedral arrangement of water molecules, which is characteristic of the hydrogen-bond network of the ambient water structure, collapses at temperatures in excess of $416 \mathrm{~K}^{25,42,46}$ In addition, the number of hydrogen bonds per molecule decreases from about 3.6 at ambient temperature to about $1.5-2$ at around the critical temperature. ${ }^{24-29,39}$ Thus the average structure of hightemperature water has been elucidated to a certain extent. However, molecular level behavior of high-temperature water has not yet been clarified to a satisfactory level. Moreover, the molecular-level structure of aqueous mixtures at high temperatures and pressures still remains largely unknown, although the thermodynamic properties have been widely studied. Infrared spectroscopy is one of the most useful techniques to study the properties of aqueous systems at the molecular level.

In situ infrared measurements provide quantitative information about the composition of mixtures at high temperatures and pressures. This is a particularly useful technique for the study of each of the two coexisting phases. Recently, we analyzed a hydrocarbon-rich phase of water-hydrocarbon mixtures at high temperatures and pressures, and obtained the concentrations of water and hydrocarbons as a function of temperature and pressure. ${ }^{51,52} \mathrm{We}$ discovered that the water concentration, estimated from the integrated intensities of the $\mathrm{OH}$ stretching bands, shows strong pressure dependence in the region around an extended line of the three-phase coexistence curve in the phase diagram. Moreover, the waterhydrocarbon mixtures exhibit anomalous volumetric behavior in the vicinity of the critical region. ${ }^{53}$ For example, volume expansion on the mixing of water and benzene becomes as large as $200 \%$ at $573 \mathrm{~K}$ and 100 bar. Similar phenomena have been observed for water-toluene, waterethylbenzene, and water-hexane mixtures. ${ }^{54}$ Thus the anomalous volume expansion is considered to be common to the mixing of hydrophobic hydrocarbons and water in the vicinity of the critical region. These findings are for the hydrocarbon-rich phase in the two-phase coexistence region. We were interested in establishing whether the water-rich phase in the same region shows the corresponding volumetric behavior. To examine this, the concentrations of both water and hydrocarbons in the water-rich phase are required. However, we were unable to measure the concentrations in the water-rich phase due to the strong infrared absorption of water. We then attempted to measure the concentrations using near infrared absorption caused by the overtone $\mathrm{OH}$ stretching transition.

Measurement of the overtone absorption has some advantage over the fundamental absorption. Intensity of the overtone band is two orders of magnitude smaller than the fundamental $\mathrm{OH}$ stretching band, ${ }^{30,31,55-58}$ and the integrated intensity can be obtained with an optical pathlength of about $1 \mathrm{~mm}$, which is in the range that can be controlled reasonably easily and accurately even at high temperatures and pressures. Furthermore, the effect of hydrogen bonding on the absorption intensity of the overtone band is much milder than that on the fundamental band. ${ }^{55,56}$ It is well established that the integrated intensity of the fundamental $\mathrm{OH}$ stretching band increases remarkably with increasing hydrogen bond strength. ${ }^{59}$ For example, the molar absorption intensity of the water molecule increases by an order of magnitude from the gas to the liquid phase. ${ }^{60}$ In contrast, the molar absorption intensity of the overtone band shows a slight decrease with increasing hydrogen bond strength. ${ }^{55,56}$ Nevertheless, the band position shifts significantly as a result of the hydrogen bonding. Therefore, to obtain information on the state of the hydrogen bonding in fluid or liquid water, the absorption profile of the overtone band is superior to that of the fundamental band. In the latter band, a feeble hydrogenbond-free component, which would exist to an appreciable extent, is heavily buried under the strong hydrogen-bonded components. Making use of the advantages of the nearinfrared overtone absorption, it is possible to measure the spectral profile at a wide range of densities, from the gas phase to the supercritical state of a liquidlike density. In this paper, we report evolution of the band profile and the integrated intensity with increasing pressure at sub- and supercritical temperatures, and discuss the origin of the band profile and the hydrogen-bond structure of water. These data will also be useful to the study of aqueous mixtures.

\section{EXPERIMENT}

\section{A. Apparatus and procedure}

Figure 1 shows the schematic of the high-pressure cell, which is a custom-made cell machined by Teramecs Co. (Kyoto, Japan). The cell body is made of Hastelloy, which is a nickel-base superalloy and useful for applications requiring high strength and corrosion resistance up to $1400 \mathrm{~K}$. The maximum operating temperature and pressure of the cell are $773 \mathrm{~K}$ and 500 bar, respectively. The windows of the cell are colorless sapphire with an effective aperture for optical transmission of $6 \mathrm{~mm}$, and are pressed against optically polished flat surfaces of window plugs for a pressure seal by the unsupported area principle. The optical path length of a sample is $1.46 \mathrm{~mm}$, which was determined by comparison of the absorption intensity of a near infrared band of water at room temperature with that measured using an ordinary liquid cell of known path length. The cell is heated by rod-shape electric heaters that are inserted into holes bored in the cell body, and the sample temperature is measured with a chromelalumel thermocouple. The cell has three sample inlets. The one on the top is for initial filling of sample liquids and the two on the side are for transmitting compressed liquids into the cell with a syringe pump for liquid chromatography. The upper one of the two side inlets is for hydrocarbons and the lower one for water. Pressure of the sample is measured with a pressure transducer of a semiconductor strain gage, which is set between the cell and the pump.

Near-infrared transmission spectra were measured with a Perkin-Elmer System 2000 Fourier-transform spectrometer, which was equipped with a near-infrared source and a nearinfrared detector. Spectral measurements were performed 


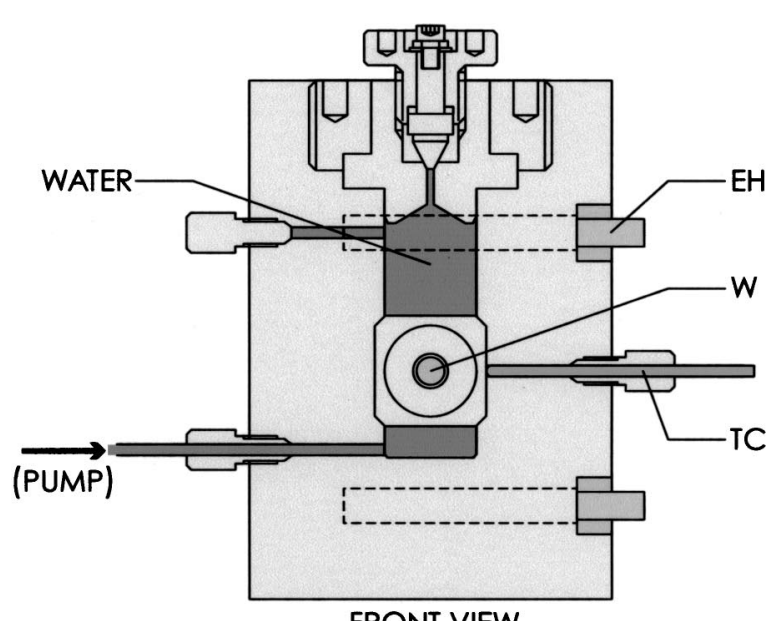

FRONT VIEW

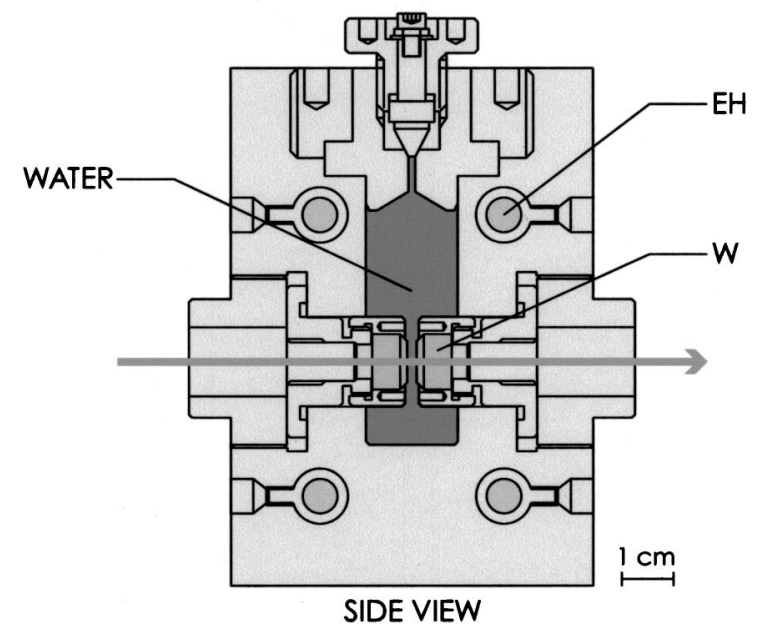

FIG. 1. Schematic of the high-pressure-high-temperature cell. EH, electric heater; W, sapphire windows; TC, thermocouple.

with $2 \mathrm{~cm}^{-1}$ resolution at sample temperatures in the 373$673 \mathrm{~K}$ range and pressures in the 20-400 bar range. Each of the experimental temperature-pressure points was attained at a slow rate, and the phase equilibrium of sample fluid was confirmed by a spectrum that remained unchanged for at least an hour.

\section{B. Observed spectra}

Figure 2 illustrates how the absorption profile of the near-infrared $\mathrm{OH}$-stretching overtone transition varies with increasing temperature from 293 to $673 \mathrm{~K}$ at a constant pressure of 400 bar. The absorbance has been normalized by densities $\left(\mathrm{g} / \mathrm{cm}^{3}\right)$ which are taken from NIST Chemistry WebBook. ${ }^{61}$ As the temperature rises, the absorption in the region below $7000 \mathrm{~cm}^{-1}$ decays rapidly while the absorption at higher wave numbers grows remarkably. At the low-wavenumber end of the figure, a tail of another band appears, which is centered around $5200 \mathrm{~cm}^{-1}$ and assigned to an $\mathrm{OH}$ stretching + bending combination transition. The absorption of this band is several times stronger than the $\mathrm{OH}$ overtone band concerned in the present study. The lower-wavenumber tail of the overtone band is thought to originate from the hydrogen bond network, in which the $\mathrm{OH}$ groups exhibit broad absorption at lower wave numbers due to cooperativity

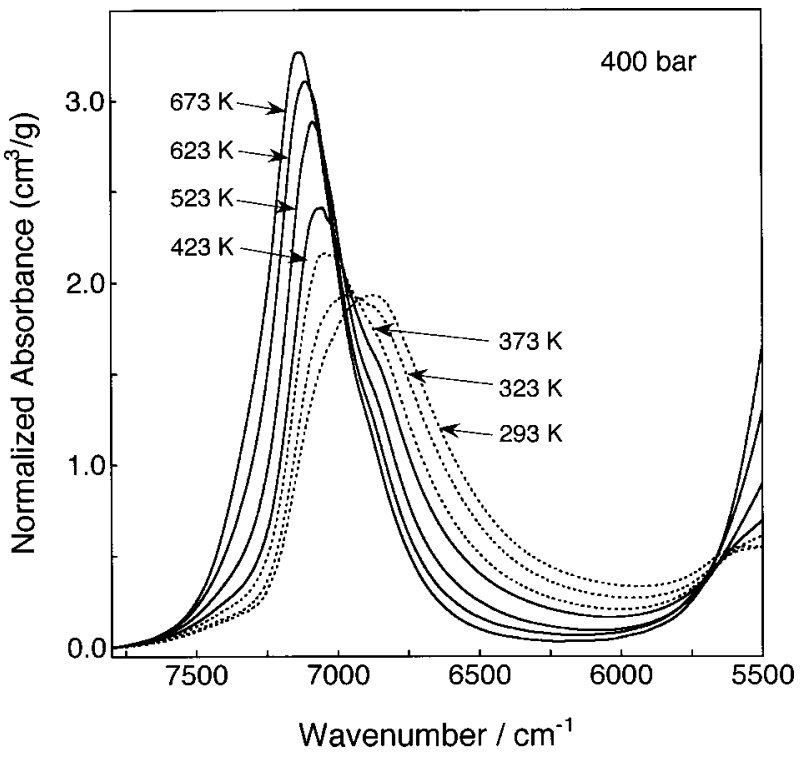

FIG. 2. Effect of temperature on the absorption profile of the near-infrared $\mathrm{OH}$ stretching overtone band of water at 400 bar. The absorbance has been normalized by densities $\left(\mathrm{g} / \mathrm{cm}^{3}\right)$.

of the hydrogen bonds. ${ }^{62}$ Therefore, the rapid decay of absorption in the 6800 to $6000 \mathrm{~cm}^{-1}$ region may be related to a reduction in, or fragmentation of the hydrogen bond network in water. This is consistent with the structural change observed by $\mathrm{x}$-ray ${ }^{25}$ and MD simulation studies, ${ }^{42,46}$ which is characterized by decay of the quasitetrahedral hydrogen bonded structure.

A small bulge at around $6850 \mathrm{~cm}^{-1}$, which appears above $373 \mathrm{~K}$, decays with rising temperature and is assigned to hydrogen bonded $\mathrm{OH}$ groups. However, it seems to persist even at $673 \mathrm{~K}$, and may be assigned to small hydrogenbonded clusters, such as dimers and trimers, being consistent with the previous infrared study of the fundamental $\mathrm{OH}$ stretching region. ${ }^{34}$ The absorption in the region above 7000 $\mathrm{cm}^{-1}$ is attributed to hydrogen-bond-free $\mathrm{OH}$ groups. The peak maximum gradually shifts to higher wave numbers, and a slight bump emerges at around $7400 \mathrm{~cm}^{-1}$ at higher temperatures. To further characterize these band features, it is useful to examine the evolution of the band shape with increasing pressure at a constant temperature. ${ }^{32,34,35}$

Figure 3 displays the observed spectra of water at pressures in the 20 to 400 bar range at a constant temperature of $673 \mathrm{~K}$, where an inlet figure shows the spectra at lower pressures on an expanded scale. The rotational fine structure, which is clearly observed in spectra at the lowest two pressures, gradually collapses to a smooth envelope with $P, Q$, and $R$ branches as the pressure increases. At the higher pressures, the $P, Q$, and $R$ branches merge into a single band. However, the $R$ branch does not completely fade even at 400 bar, but remains as a slight bump on the higher-wave-number side of the band. A feature of the $P$ branch cannot be seen at the higher pressures, due to faster mergence with the $Q$ branch and overlap with the growing absorption of hydrogen-bonded $\mathrm{OH}$ groups on the lower-wave-number side. Thus the absorption band still retains rotational features to a certain extent at $673 \mathrm{~K}$ and $400 \mathrm{bar}$, where the density is 


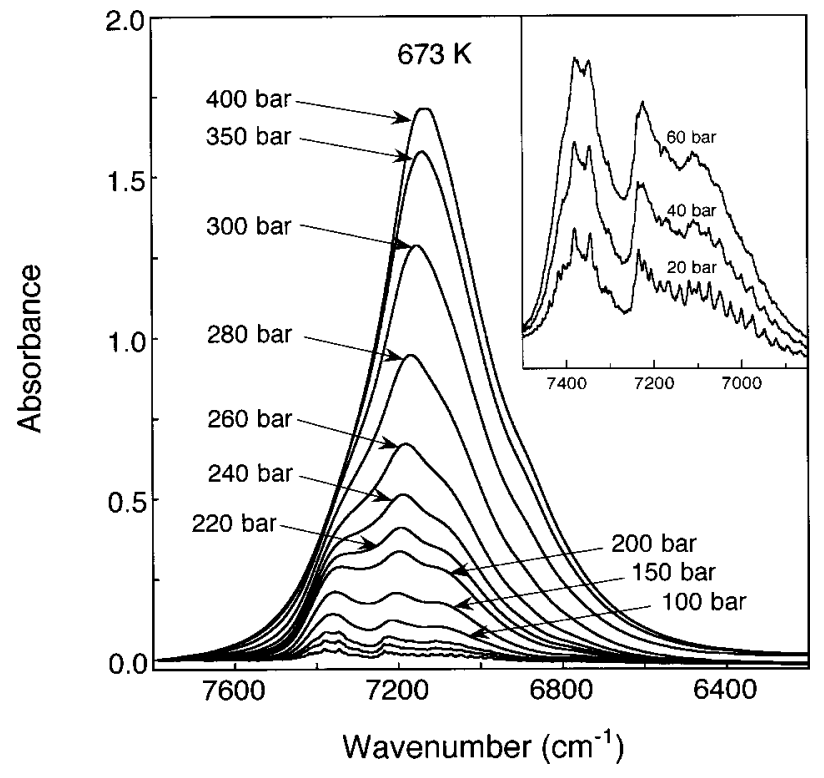

FIG. 3. Effect of pressure on the near-infrared $\mathrm{OH}$ stretching overtone absorption of water at $673 \mathrm{~K}$.

$29 \mathrm{~mol} / \mathrm{l}$. This fact indicates that an appreciable proportion of the molecules rotate quite freely, although they undergo frequent collisions with other molecules. The spectra of water have usually been decomposed to symmetric components with Lorentzian and/or Gaussian profiles to estimate the ratios of the different hydrogen-bonded states of water molecules. ${ }^{31,36,56,58}$ However, such a method is not useful for analysis of the present spectrum. A contribution of the free molecules exhibiting the remnant of the $P, Q$, and $R$ branches is difficult to separate from the heavily overlapping components bands that are related to different hydrogenbonded states. Therefore, to discuss the state of hydrogen bonding in water, we have estimated the integrated intensity and the first moment of the absorption bands.

\section{DISCUSSION}

The integrated intensities of the $\mathrm{OH}$ stretching overtone absorption bands have been estimated in the frequency range of 6200 to $7800 \mathrm{~cm}^{-1}$. The molar absorption intensities are obtained from the integrated intensities divided by the molar concentration of water $C_{w}$ taken from the literature: ${ }^{61}$

$$
A=\frac{1}{C_{w} l} \int \ln \left(I_{0} / I\right) d \nu,
$$

where $l$ denotes the sample thickness, $0.146 \mathrm{~cm}$, and $I$ and $I_{0}$ transmission spectra of the cell with and without sample, respectively. Reflection at a fluid-window interface should influence the $I$ and $I_{0}$ spectra in different ways and yield a slight, nearly vertical shift in the absorbance. To exclude this effect from the integration, a horizontal base line is taken at a signal level of $7800 \mathrm{~cm}^{-1}$, where the overtone absorption decays sufficiently. The lower frequency limit of the integration range, $6200 \mathrm{~cm}^{-1}$, is set to allow for the overlapping with the lower-frequency combination band. This may yield some error in the estimation of the absolute values of the integrated intensity at the lower temperatures or at the higher

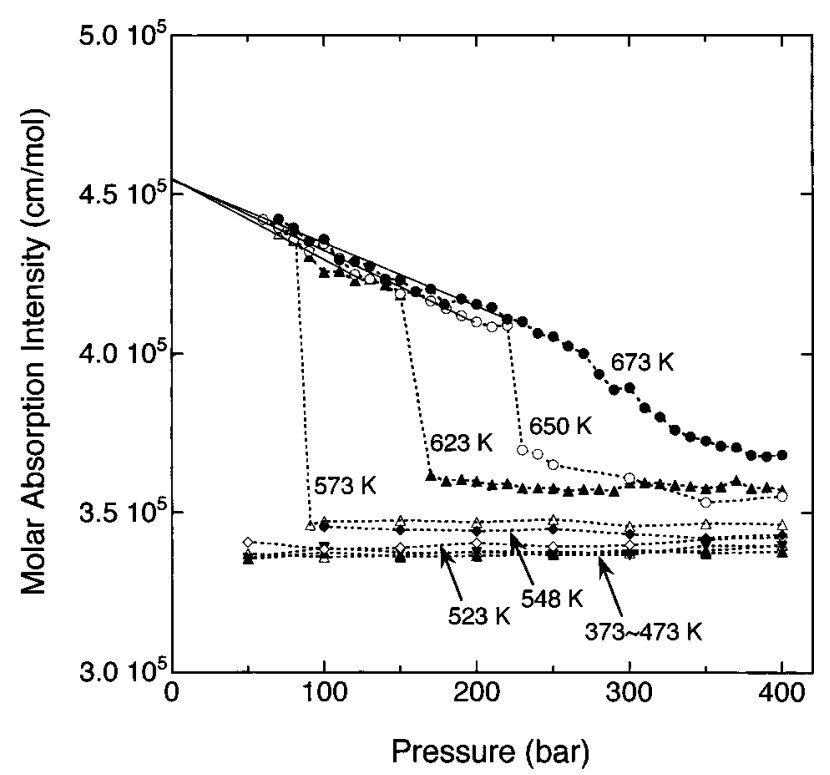

FIG. 4. Plots of molar absorption intensity of the near-infrared $\mathrm{OH}$ stretching overtone transition of water against pressure at various temperatures.

densities, but will not affect the following discussion. For spectra recorded at pressures below 50 bar that exhibit a distinct rotational fine structure, the intensities were inaccurate due to uncertainty in the position of the baseline and were excluded from the following discussion.

The resulting molar absorption intensities are plotted against pressure in Fig. 4. The discrete jumps at 573 and 623 $\mathrm{K}$ are due to the gas-to-liquid transition. At $650 \mathrm{~K}$, which is slightly above the critical temperature, the intensities are extremely sensitive to pressure at around the critical point and we have obtained a quasidiscrete jump. However, at $673 \mathrm{~K}$, the intensities exhibit a large but smooth variation with pressure. The decrease of the molar absorption intensities with rising pressure is attributed to the effect of hydrogen bonding between water molecules. This is in contrast to the pressure dependence of the $\mathrm{OH}$ stretching fundamental absorption intensity, which shows a remarkable increase due to hydrogen bonding. ${ }^{32,33}$ Thus the effect of hydrogen bonding on the molar absorption intensity is opposite for the fundamental and the overtone $\mathrm{OH}$ stretching transitions.

The approximately linear decrease of the intensity in the low-pressure region, seen in Fig. 4, is attributed to a pressure-induced shift of the monomer-dimer equilibrium. The observed molar absorption intensity is given by an average of those of the free molecules, $A_{m}$, and the dimers, $A_{d}$,

$$
A=(1-x) A_{m}+x A_{d},
$$

where $x$ denotes a mole fraction of water molecules forming the dimers. It is well established that the water dimer has a translinear configuration, which consists of a hydrogendonor and a hydrogen-acceptor molecule. ${ }^{63-65}$ Therefore, the value of $A_{d}$ is considered to be an average for these two different states of molecules in the dimer:

$$
A_{d}=\left(A_{d d}+A_{d a}\right) / 2 .
$$

The equilibrium constant is given by 


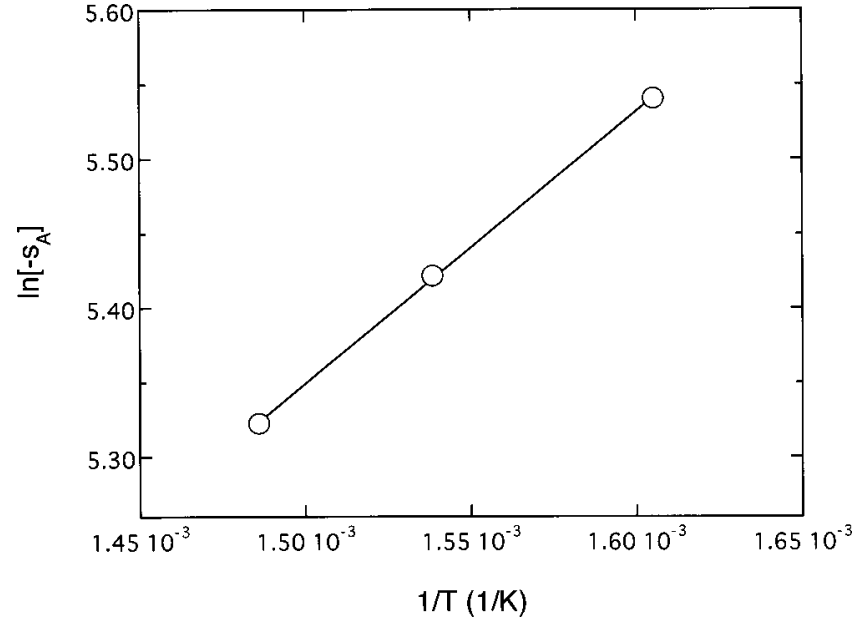

FIG. 5. van't Hoff plots for dimerization of water in the gas phase.

$$
K=\frac{x(2-x)}{4(1-x)^{2} P},
$$

where $P$ denotes the total pressure. It is easily shown that $x$ is approximately equal to $2 K P$ in the range of $x \ll 1$. Then

$$
A=A_{m}+2 K P\left(A_{d}-A_{m}\right) .
$$

Thus the observed negative slope of the plots of $A$ against pressure $P$ indicates $A_{d}<A_{m}$. Least squares fitting was performed for the plots of the three higher temperatures with constraint of the same intercept. A value of $A_{m}=(4.55$ $\pm 0.02) \times 10^{5} \mathrm{~cm} / \mathrm{mol}$ is obtained from the intercept. Since $A_{m}$ and $A_{d}$ are constant, the temperature dependence of the slope, $s_{A}=2 K\left(A_{d}-A_{m}\right)$, provides enthalpy change for the dimerization,

$$
\Delta H=-R \frac{\partial \ln K}{\partial(1 / T)}=-R \frac{\partial \ln \left[-s_{A}\right]}{\partial(1 / T)},
$$

where $R$ is the gas constant. Figure 5 shows plots of $\ln$ $\left[-s_{A}\right]$ against inverse temperature, and the slope of these plots gives a $\Delta H$ value of $15 \pm 3 \mathrm{~kJ} \mathrm{~mol}^{-1}$. This is in good agreement with the previously reported result, 16.65 \pm 3.77 $\mathrm{kJ} \mathrm{mol}^{-1}$, which was obtained from analysis of the absorption intensity of the $\mathrm{OH}$ stretching fundamental transition. ${ }^{33}$

At present, the equilibrium constant $K$ cannot be experimentally determined, since the value of $A_{d}$ is unavailable. It appears difficult to measure an $A_{d}$ value in the gas phase. Nevertheless, a rough estimation of $A_{d}$ may be possible by use of a matrix isolation study and theoretical calculations, so we attempted to estimate the fraction of water molecules forming the dimers in the compressed gases. Recently, Perchard ${ }^{66}$ reported measurements of infrared intensities of the fundamental and overtone bands of water trapped in nitrogen matrix and performed detailed analysis of the intensities of various transitions. The results have indicated that the molar absorption intensity of the overtone transition of the proton acceptor molecule in the dimer is approximately equal to that of the monomer, $A_{d a} \cong A_{m}$. For the proton donor molecule, the molar absorption intensity originates predominantly from the $\mathrm{OH}$ bond that is free from hydrogen bonds, while the other $\mathrm{OH}$ bond, forming the hydrogen-bond

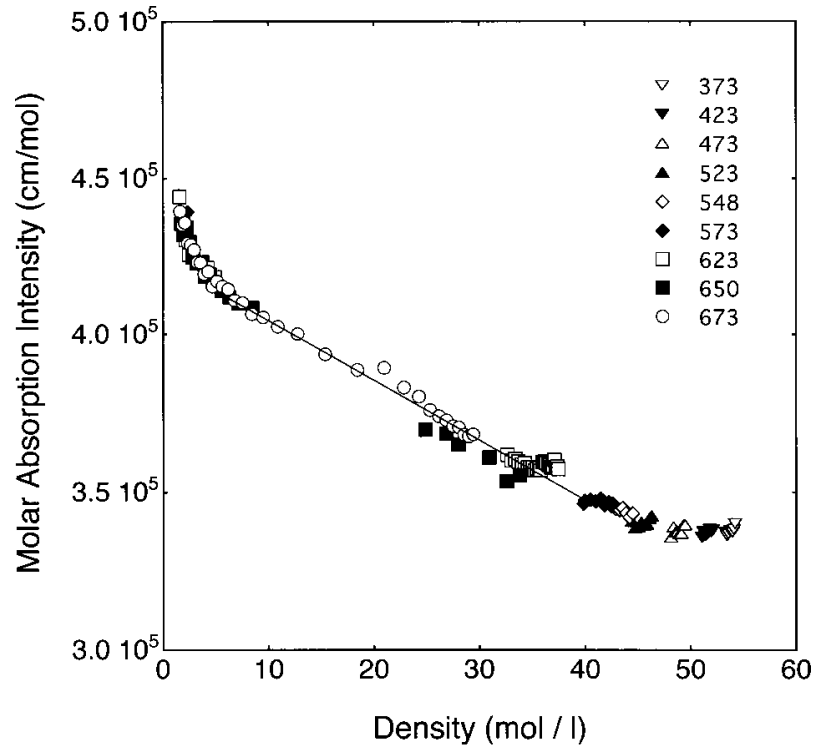

FIG. 6. Plots of molar absorption intensity of the near-infrared $\mathrm{OH}$ stretching overtone transition of water against molar density.

bridge, contributes a negligibly small intensity to the overtone transition. This is in marked contrast with their contribution to the fundamental $\mathrm{OH}$ stretching transition. These experimental results in nitrogen matrix are consistent with $a b$ initio theoretical calculations of water dimer by Low and Kjaergaard. ${ }^{67}$ Furthermore, these experimental and theoretical results indicate that the molar absorption intensity of the proton donor molecule is roughly half that of the monomer, $A_{d d} \cong A_{m} / 2$. By use of these estimations, Eq. (5) can be reduced to the following form:

$$
A=A_{m}(1-K P / 2) .
$$

Therefore, the equilibrium constant $K$ is given by the ratio of the slope to the intercept in the plots. The resulting values are $9 \times 10^{-4}, 10 \times 10^{-4}$, and $11 \times 10^{-4} \mathrm{bar}^{-1}$ at 673,650 , and $623 \mathrm{~K}$, respectively, and the molar fraction of dimerization, $x$, is estimated to be $0.18,0.20$, and 0.22 , at 100 bar. Therefore, roughly $20 \%$ of water molecules take part in the dimerization at 100 bar in the above temperature range.

Figure 6 shows plots of the molar absorption intensity versus molar density. The plots at different temperatures are approximately on the same line in the whole density region. The rate of decrease in the molar absorption with increasing density exhibits a change at around $2 \mathrm{~mol} / \mathrm{l}$. The steep decrease at the lower densities is attributed to the dimerization mentioned above. In the middle density range, from 6 to 40 $\mathrm{mol} / \mathrm{l}$, the molar absorption decreases almost linearly even when passing the vapor-to-liquid transition. In the region of the higher densities, the molar absorption appears to converge. These facts indicate that the ratio of the hydrogenbond formation is largely dependent on the molar density only, irrespective of the temperature in the temperature range concerned. This can be understood by considering that the energy of the hydrogen bonding of water molecules, about $20 \mathrm{~kJ} / \mathrm{mol}$, is distinctly higher than the average thermal energy, 5 to $6 \mathrm{~kJ} / \mathrm{mol}$ in the $573-673 \mathrm{~K}$ range. 


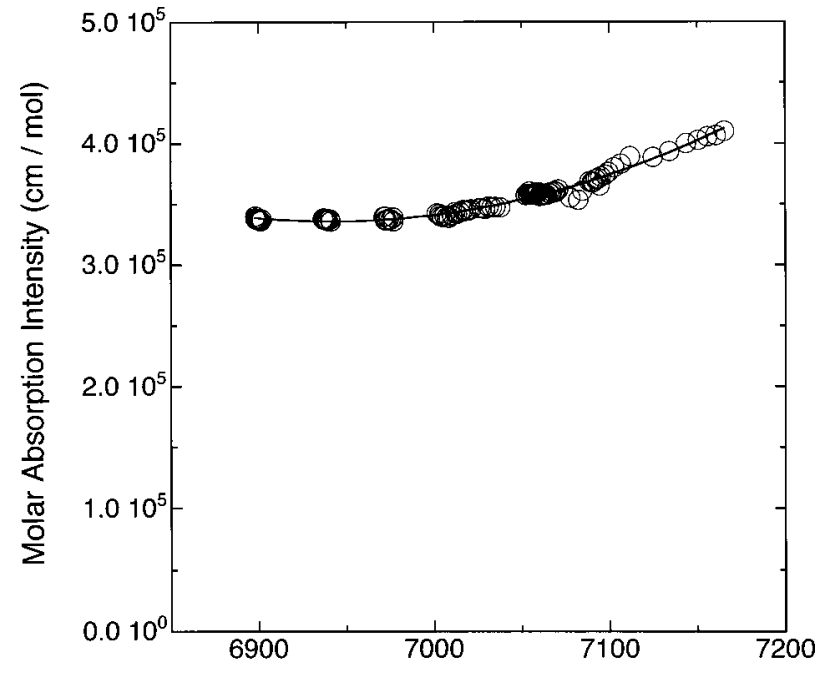

First Moment $\left(\mathrm{cm}^{-1}\right)$

FIG. 7. Correlation between molar absorption intensity and the first moment of the near-infrared $\mathrm{OH}$ stretching overtone transition.

In addition to the molar absorption intensity of the $\mathrm{OH}$ stretching transition, the wave number of the band center provides another measure of the extent of hydrogen bonding. It is well known that the band center shifts to higher wave number as the degree of hydrogen bonding decreases. Since the overtone band concerned in the present study is not symmetrical, we take the first moment of the band

$$
\bar{\nu}=\int \nu \ln \left(I_{0} / I\right) d \nu / \int \ln \left(I_{0} / I\right) d \nu,
$$

as the center of gravity of the absorption band. The resulting first moments at densities in liquid and liquid-like regions show a good correlation with the molar absorption intensities, as seen in Fig. 7. The relationship between the first moment and the molar absorption intensity will be useful for the study of aqueous mixtures. Since both the quantities are mainly determined by the hydrogen-bonding state of water molecules, we may assume that the relationship given by Fig. 7 can be applied to aqueous mixtures in which the molar absorption intensity is determined by the hydrogen-bonding state. From the first moment of the band, which can be obtained from the observed absorption without knowledge of concentration, the molar absorption intensity is given by the relationship. Then the water concentration in the aqueous mixtures can be estimated from the observed absorption intensity divided by the molar absorption intensity. This spectroscopic method is particularly useful to estimate the water concentration in one phase of the two-phase coexistence region of aqueous mixtures such as water-hydrocarbon mixtures at high temperatures and pressures, where only in situ spectroscopic measurements provide reliable information without disturbing the equilibrium.

A plot similar to Fig. 7 has been reported for the fundamental OD stretching absorption of HDO diluted in $\mathrm{H}_{2} \mathrm{O} .{ }^{32}$ The molar absorption intensity of the fundamental transition decreases by an order of magnitude as the band peak position shifts by about $160 \mathrm{~cm}^{-1}$. In marked contrast to this, the molar absorption intensity of the overtone transition increases by only $20 \%$ as the first moment increases by 270 $\mathrm{cm}^{-1}$, as illustrated in Fig. 7. This is an advantage of using the overtone band to estimate water concentration in aqueous mixtures, because uncertainty in the estimates of the molar absorption intensity is quite small and the water concentration can be estimated without significant uncertainty. The relationship given by Fig. 7 will be used in a study of waterbenzene mixtures at high temperatures and pressures in the next paper.

\section{ACKNOWLEDGMENTS}

The authors thank Dr. Seiya Furutaka for the valuable discussions. They also thank Dr. Takuya Fukuda for his kind support in use of the near-infrared light source. This work was supported by the Grant-in-Aid for Scientific Research from the Ministry of Education, Culture, Sports, Science and Technology of Japan (No. 13440169).

${ }^{1}$ W. H. Thompson and J. R. Snyder, J. Chem. Eng. Data 9, 516 (1964).

${ }^{2}$ J. F. Connolly, J. Chem. Eng. Data 11, 13 (1966).

${ }^{3}$ Z. Alwani and G. M. Schneider, Ber. Bunsenges. Phys. Chem. 71, 633 (1967).

${ }^{4}$ S. L. Wallen, B. J. Palmer, D. M. Pfund, J. L. Fulton, M. Newville, Y. Ma, and E. A. Stern, J. Phys. Chem. A 101, 9632 (1997).

${ }^{5}$ O. Kritzer, N. Boukis, and E. Dinjus, J. Supercrit. Fluids 15, 205 (1999).

${ }^{6}$ R. A. Mayanovic, S. Jayanetti, A. J. Anderson, W. A. Basett, and I.-M. Chou, J. Chem. Phys. 118, 719 (2003).

${ }^{7}$ D. G. Archer and P. Wang, J. Phys. Chem. Ref. Data 19, 371 (1990).

${ }^{8}$ K. Krynicki, C. D. Green, and D. W. Sawyer, Faraday Discuss. Chem. Soc. 66, 199 (1979).

${ }^{9}$ W. J. Lamb, G. A. Hoffman, and J. Jonas, J. Chem. Phys. 74, 6875 (1981).

${ }^{10}$ A. R. Katritzky, D. A. Nicols, M. Siskin, R. Murugan, and M. Balasubramanian, Chem. Rev. 101, 837 (2001).

${ }^{11}$ N. Akiya and P. E. Savage, Chem. Rev. 102, 2725 (2002).

${ }^{12}$ J. W. Tester and J. A. Cline, Corrosion (Houston) 55, 1088 (1999).

${ }^{13}$ P. Kritzer and E. Dinjus, Chem. Eng. J. 83, 207 (2001).

${ }^{14}$ Th. Hirth and E. U. Franck, Ber. Bunsenges. Phys. Chem. 97, 1091 (1993).

${ }^{15}$ I. Hua, R. H. Hochemer, and M. R. Hoffmann, J. Phys. Chem. 99, 2335 (1995).

${ }^{16}$ T. Sako, T. Sugeta, K. Otake, M. Sato, M. Tsugumi, T. Hiaki, and M. Hongo, J. Chem. Eng. Jpn. 30, 744 (1997).

${ }^{17}$ Y. Yamasaki, H. Enomoto, N. Yamasaki, and M. Nakahara, Bull. Chem. Soc. Jpn. 73, 2687 (2000).

${ }^{18}$ J. S. Rowlinson and F. L. Swinton, Liquids and Liquid Mixtures, 3rd ed. (Butterworth, London, 1982).

${ }^{19}$ C. Tsonopoulos and G. M. Wilson, AIChE J. 29, 990 (1983).

${ }^{20}$ C. J. Wormald, Ber. Bunsenges. Phys. Chem. 88, 826 (1984).

${ }^{21}$ J. Li, I. Vanderbeken, S. Ye, H. Carrier, and P. Xans, Fluid Phase Equilib. 131, 107 (1997).

${ }^{22}$ P. Postorino, R. H. Tromp, M. A. Ricci, A. K. Soper, and G. W. Neilson, Nature (London) 366, 668 (1993).

${ }^{23}$ A. K. Soper, F. Bruni, and M. A. Ricci, J. Chem. Phys. 106, 247 (1997).

${ }^{24}$ M.-C. Bellissent-Funel, T. Tassaing, H. Zhao, D. Beysens, B. Guillot, and Y. Guissani, J. Chem. Phys. 107, 2942 (1997).

${ }^{25}$ K. Yamanaka, T. Yamaguchi, and H. Wakita, J. Chem. Phys. 101, 9830 (1994).

${ }^{26}$ Yu. E. Gorbaty and A. G. Kalinichev, J. Phys. Chem. 99, 5336 (1995).

${ }^{27}$ M. H. Hoffmann and M. S. Conradi, J. Am. Chem. Soc. 119, 3811 (1997).

${ }^{28}$ N. Matubayasi, C. Wakai, and M. Nakahara, Phys. Rev. Lett. 78, 2573 (1997).

${ }^{29}$ N. Matubayasi, C. Wakai, and M. Nakahara, J. Chem. Phys. 107, 9133 (1997).

${ }^{30}$ W. A. P. Luck, Ber. Bunsenges. Phys. Chem. 69, 626 (1965).

${ }^{31}$ W. A. P. Luck and W. Ditter, Z. Naturforsch. B 24, 482 (1969).

${ }^{32}$ E. U. Franck and K. Roth, Discuss. Faraday Soc. 43, 108 (1967).

${ }^{33}$ G. V. Bondarenko and Yu. E. Gorbaty, Mol. Phys. 74, 639 (1991). 
${ }^{34}$ T. Tassaing, Y. Danten, and M. Besnard, J. Mol. Liq. 101, 149 (2002).

${ }^{35}$ W. Kohl, H. A. Lindner, and E. U. Franck, Ber. Bunsenges. Phys. Chem. 95, 1586 (1991).

${ }^{36}$ D. M. Carey and G. M. Korenowski, J. Chem. Phys. 108, 2669 (1998).

${ }^{37}$ Y. Ikushima, K. Hatakeda, and N. Saito, J. Chem. Phys. 108, 5855 (1998).

${ }^{38}$ G. E. Walrafen, W.-H. Yang, and Y. C. Chu, J. Phys. Chem. B 103, 1332 (1998); 105, 7155 (2001).

${ }^{39}$ S. J. Suresh and V. M. Naik, J. Chem. Phys. 113, 9727 (2000).

${ }^{40}$ R. D. Mountain, J. Chem. Phys. 90, 1866 (1989).

${ }^{41}$ P. T. Cummings, H. D. Cochran, J. M. Simonson, R. E. Mesmer, and S. Karaborni, J. Chem. Phys. 94, 5606 (1991).

${ }^{42}$ Y. Guissani and B. Guillot, J. Chem. Phys. 98, 8221 (1993).

${ }^{43}$ G. Loffler, H. Schreiber, and O. Steinhauser, Ber. Bunsenges. Phys. Chem. 98, 1575 (1994).

${ }^{44}$ A. A. Chialvo and P. T. Cummings, J. Chem. Phys. 101, 4466 (1994).

${ }^{45}$ T. I. Mizan, P. E. Savaga, and R. M. Ziff, J. Phys. Chem. 100, 403 (1996).

${ }^{46}$ P. Jedlovszky, J. P. Brodholt, F. Bruni, M. A. Ricci, A. K. Soper, and R. Vallauri, J. Chem. Phys. 108, 8528 (1998).

${ }^{47}$ N. Yosii, H. Yosie, S. Miura, and S. Okazaki, J. Chem. Phys. 109, 4873 (1998).

${ }^{48}$ J. Marti, J. Chem. Phys. 110, 6876 (1999).

${ }^{49}$ B. Guillot and Y. Guissani, J. Chem. Phys. 114, 6720 (2001).

${ }^{50}$ B. Guillot, J. Mol. Liq. 101, 219 (2002).

${ }^{51}$ S. Furutaka and S. Ikawa, J. Chem. Phys. 113, 1942 (2000).

${ }^{52}$ S. Furutaka, H. Kondo, and S. Ikawa, Bull. Chem. Soc. Jpn. 74, 1775 (2001).
${ }^{53}$ S. Furutaka and S. Ikawa, J. Chem. Phys. 117, 1682 (2002).

${ }^{54}$ S. Furutaka and S. Ikawa, Fluid Phase Equilib. (in press).

${ }^{55}$ J.-J. Peron, C. Bourderon, and C. Sandorfy, Can. J. Chem. 49, 3901 (1971).

${ }^{56}$ G. R. Choppin and M. R. Violante, J. Chem. Phys. 56, 5890 (1972).

${ }^{57}$ V. H. Segtnan, S. Sasic, T. Isaksson, and Y. Ozaki, Anal. Chem. 73, 3153 (2001).

${ }^{58}$ D. E. Khoshtariya, T. D. Dolidze, P. Lindqvist, A. Neubrand, and R. van Eldik, J. Mol. Liq. 96-97, 45 (2003).

${ }^{59}$ G. C. Pimentel and A. L. McClellan, The Hydrogen Bond (Freeman, San Francisco, 1960).

${ }^{60}$ S. Ikawa and S. Maeda, Spectrochim. Acta, Part A 24, 655 (1968).

${ }^{61}$ E. W. Lemmon, M. O. McLinden, and D. G. Friend, in NIST Chemistry WebBook, NIST Standard Reference Database Number 69, edited by P. J. Linstrom and W. G. Mallard (National Institute of Standards and Technology, Gaithersburg, MD, 2001) (http://webbook.nist.gov).

${ }^{62}$ W. A. P. Luck, J. Mol. Struct. 448, 131 (1998).

${ }^{63}$ T. R. Dyke, K. M. Mack, and J. S. Muenter, J. Chem. Phys. 66, 498 (1977).

${ }^{64}$ O. Matsuoka, E. Clementi, and M. Yosimine, J. Chem. Phys. 64, 1351 (1976).

${ }^{65}$ Z. S. Huang and R. E. Miller, J. Chem. Phys. 91, 6613 (1989).

${ }^{66}$ J. P. Perchard, Chem. Phys. 266, 109 (2001).

${ }^{67}$ G. R. Low and H. G. Kjaergaard, J. Chem. Phys. 110, 9104 (1999). 